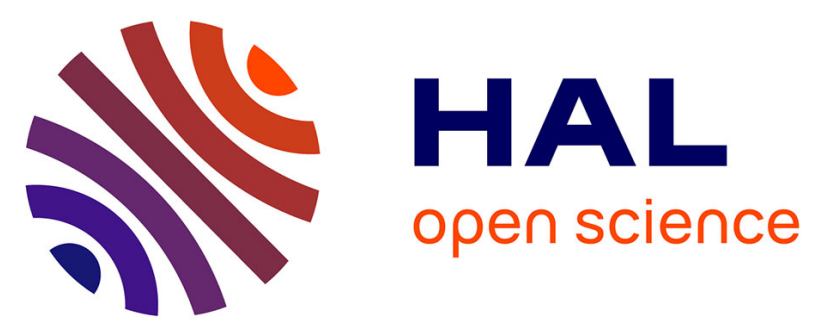

\title{
Evidence for the formation of distorted nanodomains involved in the phase transformation of stabilized zirconia by coupling convergent beam electron diffraction and in situ TEM nanoindentation
}

Emilie Calvié, Lucile Joly-Pottuz, Claude Esnouf, Thierry Douillard, Laurent

Gremillard, Annie Malchere, Jérome Chevalier, Karine Masenelli-Varlot

\section{To cite this version:}

Emilie Calvié, Lucile Joly-Pottuz, Claude Esnouf, Thierry Douillard, Laurent Gremillard, et al.. Evidence for the formation of distorted nanodomains involved in the phase transformation of stabilized zirconia by coupling convergent beam electron diffraction and in situ TEM nanoindentation. Acta Materialia, 2013, 61 (1), pp.174-182. 10.1016/j.actamat.2012.09.047 . hal-00760135

\section{HAL Id: hal-00760135 https://hal.science/hal-00760135}

Submitted on 13 Jun 2019

HAL is a multi-disciplinary open access archive for the deposit and dissemination of scientific research documents, whether they are published or not. The documents may come from teaching and research institutions in France or abroad, or from public or private research centers.
L'archive ouverte pluridisciplinaire HAL, est destinée au dépôt et à la diffusion de documents scientifiques de niveau recherche, publiés ou non, émanant des établissements d'enseignement et de recherche français ou étrangers, des laboratoires publics ou privés. 


\title{
Evidence for the formation of distorted nano-domains involved in the phase transformation of stabilized-zirconia by coupling Convergent Beam
}

\section{Electron Diffraction and in situ TEM nanoindentation}

\author{
E. Calvié, L. Joly-Pottuz *, C. Esnouf, T. Douillard, L. Gremillard, A. Malchère, J. Chevalier, \\ K. Masenelli-Varlot
}

INSA-Lyon, MATEIS UMR5510, 7 avenue J. Capelle, 69621 Villeurbanne Cedex, FRANCE

\begin{abstract}
The transformation of zirconia from its tetragonal to its monoclinic phase is an important feature of the zirconia system. First found to be an advantage due to its important toughening effect, it can also be very detrimental when it occurs in the framework of low-temperature degradation, particularly in the case of biomaterial applications. One way to avoid or to control this phase transformation is to understand how it initiates and more particularly the stress states that can trigger it. A new technique available inside a transmission electron microscope (TEM) seems to be particularly well suited for that type of study: Convergent Beam Electron Diffraction (CBED), a well-known technique to reveal stresses, was coupled to in situ TEM mechanical nanoindentation. The experiments reveal the presence of sheared nano-regions at grain boundaries. These could act as embryos for tetragonal-to-monoclinic phase transformations. This is an important first step in the understanding of the earliest stage of zirconia phase transformation.
\end{abstract}

Keywords: Transmission Electron Microscopy; In situ; CBED; zirconia

*Corresponding author: Lucile Joly-Pottuz

INSA-Lyon, MATEIS UMR 5510

7 Ave. Jean Capelle, 69621 Villeurbanne Cedex, FRANCE

Lucile.Joly-Pottuz@insa-lyon.fr

Tel: +33-4-72-437102; fax: +33-4-72-437930 


\section{1- Introduction:}

Ceramic materials demonstrate interesting properties and are used in a large range of applications. One of their main weakness is however their propensity to brittle failure. Zirconia was found to be very interesting because of a toughening mechanism reducing crackpropagation velocities. This mechanism is based on a tetragonal-to-monoclinic (t-m) phase transformation at the tip of cracks which leads to a $\sim 4$ vol. \% expansion of the zirconia cell volume. Cracks are thus stopped or slowed down by the resulting compressive stress field. This mechanism was first proposed by Garvie as an advantage for zirconia [1], and provides zirconia with the best mechanical properties of oxide ceramics. Nevertheless, the tetragonalmonoclinic transformation presents an important drawback as well. It occurs at the surface of zirconia components in the presence of water or moisture, and the volume expansion leads to a roughness increase, microcracking and possible delayed failure. This mechanism is called Low Temperature Degradation (LTD) $[2,3]$ and is not yet fully understood. It is well known that the $\mathrm{t}-\mathrm{m}$ phase transformation is martensitic: this was evidenced by numerous TEM studies and observations highlighting a twinning phenomenon in transformed grains [4-8]. However, the initiation of the transformation is not well characterized. An important step to better understand its mechanism is the study of local deformations (at the nanometer scale) either governing the transformation or resulting from it.

In this paper, the onset of phase transformation and consecutive deformation at the nanometer scale are identified. Convergent Beam Electron Diffraction (CBED) analyses on stabilized zirconias under strain have been performed during in situ nanoindentation inside TEM. This innovative tool has already been used successfully to follow grain rotations in ceramic composite thin foil [9], plasticity and dislocation movements in metals [10, 11] or nanoparticle behaviors [12-15] during nanoindentation, but to our knowledge never in CBED mode. 
First of all, the possibility of using CBED analysis on zirconia thin foils without nanoindentation will be considered. Then, CBED will be applied to analyze zirconia during in situ nanoindentation inside the TEM. From these results, suggestions on the very first step of nucleation and propagation of the phase transformation will be proposed.

\section{2- Experimental methods:}

The stable pure zirconia phase at room temperature is monoclinic but stabilized zirconia tetragonal phases are obtained by the addition of elements such as yttrium or cerium. In order to delay the phase transformation from tetragonal into monoclinic, two sintered, stabilized zirconia samples have been prepared: $3 \mathrm{Y}-\mathrm{TZP}$ and $12 \mathrm{Ce}-\mathrm{TZP}$. The average grain sizes are 0.3 and $1 \mu \mathrm{m}$ for $3 \mathrm{Y}-\mathrm{TZP}$ and $12 \mathrm{Ce}-\mathrm{TZP}$, respectively.

$3 \mathrm{Y}-\mathrm{TZP}$ has been stabilized in the tetragonal phase by the introduction of 3 mol. $\%$ of $\mathrm{Y}_{2} \mathrm{O}_{3}$. The powder (TZ3YS manufactured by Tosoh Company) was dispersed in water and, after homogenization, slip casted in a plaster mould. Debinding and sintering processes were then performed at 600 and $1400^{\circ} \mathrm{C}$ respectively for $5 \mathrm{~h}$.

12Ce-TZP (CEZ-12 powder manufactured by Daiichi Company), whose tetragonal phase is stabilized with $12 \mathrm{~mol}$. $\%$ of $\mathrm{CeO}_{2}$, was processed by uniaxial pressing at $200 \mathrm{MPa}$ and sintering at $1400{ }^{\circ} \mathrm{C}$ for $2 \mathrm{~h}$. In a previous work, several sample preparation techniques for TEM examinations have been tested [16]. It appears that preparation by Focused Ion Beam (FIB) is the most suitable method for carrying coupled CBED and in situ nanoindentation experiments [17]. The sample must be in a suitable geometry (to be deformed by the tip in the expected manner) and free of any phase transformation during the thin foil preparation.

The thin foil of 12Ce-TZP was prepared by the "lift out" method [18] using a ZEISS Nvision 40 Focused Ion Beam microscope. At first a $400 \mathrm{~nm}$-thick foil was made by milling (in order to avoid phase transformation [16]); then a final thinning was performed with weaker currents 
and voltages to obtain a final thickness of $120 \mathrm{~nm}$ (calculated by JEMS simulation [19] of CBED patterns in two beams condition on the sample).

The thin foil of 3Y-TZP was prepared by the "H-Bar" method [20]. The sample was cut with a wire saw and polished to a thickness of $30 \mu \mathrm{m}$. Finally, an electron transparent window (150 nm thick, estimated by EELS [16]) was thinned using a FEI STRATA Dual Beam FIB. In situ experiments were conducted using an in situ nanoindentation sample holder manufactured by Nanofactory Company. The sample movement is controlled in three dimensions by a piezoelectric tube in a precise movement as fine as $0.1 \mathrm{~nm}$ per step. The tip used for nanoindentation is stuck on the load sensor at the end of the sample holder and remains static. A wedge shaped diamond tip (1 $\mu \mathrm{m}$ long and $40 \mathrm{~nm}$ wide) was used. Its geometry is well adapted for thin foil compression because it avoids slipping between the tip and the sample. Conventional TEM, selected-area electron diffraction, CBED and in situ TEM analyses were conducted within a JEOL 2010F transmission electron microscope operating at $200 \mathrm{kV}$. Images were captured with a GATAN CCD Orius camera and analyzed with Digital Micrograph software. Movies were screen-captured with Camstudio free software.

In CBED mode, the beam was focused on the thin foil in an area close to $2 \mathrm{~nm}$ in diameter and the convergence angle (angular opening) was about 10mrad. Due to a broadening effect through the sample thickness, the illuminated area increased up to $10 \mathrm{~nm}$ at the bottom of the thin foil. Such a value has been calculated from Doig's formula [21].

The convergence of the electron beam must produce diffraction spots in the form of enlarged discs to be effective. Inside one disc (bright field more frequently), information is related to the lattice plane orientation in the Bragg diffraction condition. Each plane is represented by a dark line and so, the respective disposition and angles between lines give information on the lattice symmetry (local distortion, for instance) with a very high accuracy. Moreover, due to 
the residual aberration of the TEM lenses and the sample thickness, information inside the disc originates from the whole volume illuminated by the focused electron beam (i.e. a few nanometers). Lines are indexed using specific software such as JEMS [19] and Electron Diffraction [22].

However, the CBED patterns do not allow direct determination of the exact location of distortions. To do this, the LACBED (Large Angle Convergent Beam Electron Diffraction) mode has been used. In this mode, the CBED pattern is produced using a high defocus which allows to view, on the same pattern, the image (a grain boundary for example) and Bragg lines representing lattice planes [23].

\section{3- Results:}

Figure 1 presents a conventional bright field image observed on the 12Ce-TZP (Figure 1a). The indexation of the obtained diffraction pattern (figure 1b) reveals that the grain is fully in tetragonal phase. Thus it validates the sample preparation technique used, which minimizes the phase transformation of zirconia (no polishing before FIB milling). The zone axis on figure $1 \mathrm{~b}$ is too simple to obtain the sharpest lines in CBED condition, which is why the pattern in figure 1c has been obtained under a more complex orientation. On this CBED pattern, the solid lines represent the tetragonal structure while the thinnest dotted lines (not indexed) correspond to another crystal.

On the simulated pattern (figure 1d), it is shown that positions and angles of lines are completely reproduced (blue dotted lines on figure 1d) demonstrating that there is no distortion of the cell. However, line intensities do not well correspond to the experimental ones. For example, the $(\overline{1} \overline{2} \overline{3})_{t}$ line is very intense on the simulation although it does not appear on the experimental pattern. 
Figure 2 presents a conventional bright field image observed on the 3Y-TZP foil on an area located at $800 \mathrm{~nm}$ from the sample edge. The indexation of the selected area diffraction pattern (figure $2 \mathrm{~b}$ ) performed on the grain shows the coexistence of a monoclinic phase $(\mathrm{m})$ and two tetragonal phases ( $\mathrm{t} 1$ and $\mathrm{t} 2$ ) which have different orientations.

We notice the existence of a family of three parallel planes $\left((\overline{2} \overline{1} 4)_{m},(130)_{t 1}\right.$, and $\left.(123)_{t 2}\right)$ of similar inter-reticular distances $(1.215,1.137$ and $1.177 \AA$ A respectively in pure zirconia). According to stereographic projection work, there is no evidence of a specific crystallographic relationship between $\mathrm{m}$ and $\mathrm{t} 2$ phases. However, there are relationships between $\mathrm{m}$ and $\mathrm{t} 1$. For example, the $(100)_{t 1}$ plane is parallel to the $(101)_{m}$ plane.

On this same grain a CBED pattern has also been acquired. In the experimental pattern, the presence of diffraction Bragg lines of three crystals is due to their superposition along the beam, implying that the electron beam crosses two interfaces (heterophase boundaries). Indeed, the sample had to be tilted to get sharp Bragg lines (as for 12Ce-TZP). Figure 2c shows Bragg lines of $\mathrm{m}, \mathrm{t} 1$ and $\mathrm{t} 2$ phases with indexations: precise zone axes have been obtained from cell parameter data given by Igawa [24] available for pure zirconia. A CBED simulation of monoclinic zirconia has also been performed (figure 2d) by using JEMS software with the same cell parameters. In order to check the influence of the dopant, another simulation has been conducted using unit cell parameters extracted from a Rietveld refinement of X-ray diffractogram of the 3\% yttrium-doped zirconia initial powder. Differences between the two simulated patterns can be considered as negligible (not displayed).

The comparison of the experimental and simulated CBED patterns shows a lower contrast of experimental lines. In addition, some lines present in the simulated pattern are not visible (the $(2 \overline{1} \overline{7})_{m}$ line for example) and simulated intensities do not correspond to the experimental ones. Finally, Bragg lines from the monoclinic structure (the dotted lines on figure 2d) are 
twisted to the theoretical ones by angles of $3^{\circ}$ and $7^{\circ}$ for $(\overline{2} \overline{4} 0)_{m}$ and $(\overline{2} \overline{1} 4)_{m}$ lines respectively.

Figures 3a,b,c,d present CBED patterns obtained on 3Y-TZP when moving the electron beam across the heterophase boundary from $\mathrm{t} 1$ to $\mathrm{m}$ (see the arrow on figure 2a). A clear splitting of the $(14 \overline{3})_{t 2}$ line is observed with a spacing of $1 \operatorname{mrad}\left(0.06^{\circ}\right)$. Several causes may be considered to explain this splitting, the most common being the presence of local strains. This will be analyzed in the discussion part.

The same effect could be observed on a sample under strain. For example, on the $12 \mathrm{Ce}-\mathrm{TZP}$ sample, figures $3 \mathrm{e}, \mathrm{f}, \mathrm{g}, \mathrm{h}$ are taken from a movie acquired in CBED mode during an in situ nanoindentation test (applied strain increases from $3 \mathrm{e}$ to $3 \mathrm{~g}$, but remains in the elastic domain). All lines seem to be split, but the splitting of the $(103)_{t}$ line (with the same spacing than the $(14 \overline{3})_{t 2}$ line of 3 Y-TZP) is clearly distinguished. One can also note that the splitting is gradual (see figure $3 \mathrm{f}$, where only the upper part of the $(103)_{t}$ line is split) and reversible during the nanoindentation load removal. Indeed, at the end of the test, lines recover their original appearance (Figure 3g).

In order to locate distorted areas, LACBED patterns were obtained for the 12Ce-TZP foil. Figure 4a shows a small grain of $125 \mathrm{~nm}$ wide and $250 \mathrm{~nm}$ long at the boundary between two grains of about $1 \mu \mathrm{m}$ (the expected size for this material). Indexing of diffraction patterns carried out on these three grains shows that the central grain is a germ of monoclinic phase located at the boundary between two tetragonal grains. It can be noted that the grain boundary is significantly distorted to accommodate the increased volume generated by the phase transformation and that the created germ is not faceted.

Figures $4 b, c, d$, e and $f$ present a sequence of LACBED patterns performed at the interface between grains showed in figure $4 \mathrm{a}$. The tetragonal-tetragonal interfaces are marked by dark 
solid lines while the tetragonal-monoclinic interfaces are marked by dark, dotted lines. All Bragg lines are distorted reflecting local constraints. However, the $(\overline{2} 32)_{t 1}$ line is the most interesting. Indeed, it is distorted at the interface between the two tetragonal grains but not split (circled areas on figures $4 \mathrm{~b}$ and $\mathrm{f}$ ). Conversely, it appears partially split along the $\mathrm{t}_{1}-\mathrm{m}$ interface (circled areas on figures $4 \mathrm{c}, \mathrm{d}$ and e). The observed splitting is the same form as those shown in CBED patterns figure 3. The splitting areas show a distorted region in the shape of a strip about $50 \mathrm{~nm}$ wide (marked by the white dotted line in figure $4 \mathrm{c}$ ) along the $\mathrm{t}_{1}$ $m$ interface.

Moreover, by stereographic projection, we have determined that the monoclinic germ had specific crystallographic relationships with both tetragonal grains. The $(100)_{m}$ plane is coincident with the $(010)_{t 1}$ plane and the $(001)_{m}$ plane with the $(010)_{t 2}$ plane. In addition, $(001)_{t 1}$ and $(010)_{m}$ planes are separated by $15^{\circ}$ only.

\section{4- Discussion}

On a CBED pattern, lines represent the traces of lattice planes. Therefore, line shifts and tilts reveal distortions of the unit cell while intensity differences are assigned to shifts of atomic position in the cell.

The monoclinic cell distortion, highlighted by the line shifts on the CBED pattern of 3Y-TZP, is induced by local strains. These can be induced by residual stresses related to the anisotropy of the Coefficients of Thermal Expansion (CTE), which is more significant in the 3Y-TZP than in the $12 \mathrm{Ce}-\mathrm{TZP}$. They may also be due to the coexistence of different phases. In fact, the presence of tetragonal phases maintains the monoclinic phase in a non-equilibrium crystallographic state and therefore may slightly modify its structure. In both cases, the produced distortions may be accompanied by a slight movement of atoms that can lead, to a lesser extent, to the differences in intensity between the experimental and simulated patterns. 
The ion bombardment during the preparation could also have an impact on the results but we have shown in a previous work that ion implantation was mostly located on the top ten nanometers of the foil [16], while our observations were performed at its center.

In our case, it is likely that these differences in intensity have instead a structural explanation. Consider for example the line $(\overline{1} \overline{2} \overline{3})_{t}$ which is very intense on the simulation and not visible in the experimental pattern. Based on the wave extinction conditions given by international tables of crystallography (Wyckoff positions for $\mathrm{Zr}$ and $\mathrm{O}$ atoms are respectively $2 \mathrm{a}$ and $4 \mathrm{~d}$ of $\mathrm{P} 42 / \mathrm{nmc}$ space group), this line is signaled only by the zirconium stacking. So, it seems that $\mathrm{Zr}$ atom positions are affected. In fact, the introduction of $\mathrm{Y}_{2} \mathrm{O}_{3}$ as stabilizer may be the cause. Indeed, $\mathrm{Y}^{3+}$ and $\mathrm{Zr}^{4+}$ ions have different valences, and the introduction of a substitutional trivalent cation in the $\mathrm{Zr}^{4+}$ lattice induces the formation of oxygen vacancies in the cell that may lead, in stressed regions, to a displacement of $\mathrm{Zr}$ atoms.

Moreover, the equilibrium atomic distances $\mathrm{Zr}-\mathrm{O}$ (in monoclinic $\mathrm{ZrO}_{2}$ ) and $\mathrm{Y}-\mathrm{O}$ (in $\mathrm{Y}_{2} \mathrm{O}_{3}$ ) are respectively 2.05 [24] and $2.25 \AA$ [25]; this difference may also cause a slight displacement of atoms in the doped zirconia cell.

The same variations of Bragg line intensities are observed in the sample of $12 \mathrm{Ce}-\mathrm{TZP}$. The valences of $\mathrm{Ce}^{4+}$ and $\mathrm{Zr}^{4+}$ ions are identical, so there is no formation of oxygen vacancies. However, the difference between Ce-O (2.34 $\AA$ ) and $\mathrm{Zr}-\mathrm{O}$ distances could explain atomic displacements associated with the introduction of $\mathrm{CeO}_{2}$ as a dopant. On the other hand, no distortion of the cell is observed in this sample, which can be explained by the absence of phase transformation since the considered grain is fully in the tetragonal phase.

Finally, for both samples, the decreased contrast of observed lines compared to calculated patterns can be attributed, on one hand, to residual stress related to the natural bending of the foil, and on the other hand to the dispersion in energy of the beam (unfiltered). In addition, some lines present in simulated patterns are not visible on the experimental ones (the $(2 \overline{1} \overline{7})_{m}$ 
line in 3Y-TZP for example). This can happen with lines of high Miller indices. Indeed, they are so sensitive to the displacement fields that in presence of residual stresses, they can be widely split and therefore not visible.

Among the observations reported above, the most intriguing is arguably the splitting of some CBED lines. Several explanations are possible and discussed below. A first explanation might be that the diffraction contrast occurs under dynamic interaction conditions [26]. In our configuration, due to a bending effect during indentation, the electron path inside the sample may locally increase and induce a Bragg line splitting if the variation in path reaches $\xi_{\mathrm{g}} / 2$ (with $\xi_{\mathrm{g}}$, the extinction distance). Bragg lines in CBED patterns correspond to high order beams and consequently to higher values of $\xi_{\mathrm{g}}$ (typically $98.4 \mathrm{~nm}$ for the $(103)_{t}$ line. Based on JEMS simulation of CBED patterns in order to produce the same patterns as those obtained, the ratio between the sample thickness and $\xi_{\mathrm{g}}$ is close to 1.2 . Such a value is too small to give an explanation of the splitting due to a path variation (either the splitting is too small or the bending too high).

A second explanation would be the natural bending of the lattice planes induced by relaxations during thin foil preparation [27]. Indeed, the plane curvature can induce a splitting of all lines. In this case, however, there would be no preferential splitting depending on the probe position in the grain.

The third explanation, and in our opinion the most probable one, is that the splitting is due to local deformation of the atomic planes [28]. Remember that CBED technique involves only a nano-scaled region, at most $10 \mathrm{~nm}$ in diameter. This may be either the consequence (in the case of the $3 \mathrm{Y}-\mathrm{TZP}$ ) or the very beginning (in the case of the 12Ce-TZP) of the tetragonal-tomonoclinic phase transformation.

In the case of $3 \mathrm{Y}-\mathrm{TZP}$, the splitting is observed without nanoindentation by moving the probe from the tetragonal to the monoclinic region of the grain. The monoclinic lines are not split 
because the monoclinic region has not suffered significant local distortions but only rotations of planes (lines shift figure 2d). However, in the tetragonal phase, there is a partial (figure $3 b$ ) or total splitting (figure 3c) depending on the position of the probe showing the coexistence of distorted (probably sheared) and undistorted areas (figure 5a). We propose that at the interface, there is a distorted region induced by the strains of the previous phase transformation that acts as the nuclei of the future transformation propagation.

This is confirmed by LACBED patterns performed on the 12Ce-TZP (Figure 4). In addition to showing that the phase transformation is initiated preferentially at the grain boundary with the existence of specific crystallographic relationships between phases (as already demonstrated by Deville [29]), we have identified a zone of lattice plane deformation along the interface between the monoclinic germ and the tetragonal grain. It is important to note that Bragg lines are split only near the monoclinic phase (about $50 \mathrm{~nm}$ ), which is not the case at the interface between two tetragonal grains.

These observations highlight a type of deformation (probably shear) only involved by the tetragonal-to-monoclinic phase transformation.

The same splitting was observed in the $12 \mathrm{Ce}-\mathrm{TZP}$, but during nanoindentation. The stresses at the interfaces between phases cannot be the cause of splitting since the grain is fully tetragonal. We propose that, under strain, sheared nano-regions are formed (similar to those present at the interface between monoclinic and tetragonal phases in $3 \mathrm{Y}-\mathrm{TZP}$ and $12 \mathrm{Ce}-\mathrm{TZP})$. These regions could be embryos for tetragonal to monoclinic phase transformation (figure 5b). If the stress stops, the regions can be resorbed; but if the stress persists or increases, they may remain, grow and therefore cause the transformation. A grain transformation is visible in conventional TEM imaging during nanoindentation [16]. However, the CBED mode is so localized that if too high a constraint is applied, the evolution of the pattern is no longer possible because the area is lost. 


\section{5- Conclusion:}

By using CBED, LACBED and in situ TEM nanoindentation techniques, the very early beginning of tetragonal-to-monoclinic phase transformation in zirconia was investigated at the nanometer scale. We have determined that:

- The phase transformation is initiated preferentially at the grain boundaries, which are distorted to accommodate the increase in volume, but there are still specific crystallographic relationships between the initial phases and the new formed phase.

- $\quad$ There is a zone of lattice plane distortion (which are assumed to be shear) of about 50 $\mathrm{nm}$ along the tetragonal-monoclinic interfaces (different from those observed along the tetragonal-tetragonal interfaces). This area is unambiguously initiated by the tetragonal-to-monoclinic phase transformation and could be the starting point of its propagation.

- Under strain, the beginning of the tetragonal-to-monoclinic phase transformation consists of the formation of nanometric distorted domains (the same as those present at tetragonal-monoclinic interfaces) in the tetragonal phase that could act as nuclei for the formation of the monoclinic phase.

We have suggested a line of inquiry to understand better the first step of the zirconia phase transformation at the nano-scale. This study is important for understanding and optimizing the behavior of bulk stabilized-zirconia, particularly in biomaterial applications.

In order to go one step further and give the best description of local cell distortion, CBED simulations from a structural model are useful. In the context of zirconia transformation, the situation is highly complex, since the model must take into account several grains inside the thin foil [30]. As such, a simplification of the grain distribution geometry and crystallography is absolutely necessary. Such an approach is in progress and will complete the results described in this paper. 


\section{Acknowledgments}

The authors would like to acknowledge the CLYM (Centre Lyonnais de Microscopie

http://www.clym.fr) for the access to the JEOL 2010F and A. Schertel (Carl Zeiss NTS company GMBH) for the preparation of the thin foil by "lift-out technique". Financial support was provided by the Region Rhone-Alpes (Cluster MACODEV) and the Institut Universitaire de France.

\section{References}

[1] Garvie RC, Hannink RH, Pascoe RT. Nature 1975;258(5537):703-4.

[2] Kobayashi K, Kuwajima H, Masaki T. Solid State Ionics 1981;3-4:489-93.

[3] Chevalier J, Gremillard L, Virkar AV, Clarke DR. J Am Ceram Soc 2009;92(9):1901-20.

[4] Rühle M, Ma LT, Wunderlich W, Evans AG. Phys B+C 1988;150(1-2):86-98.

[5] Hayakawa M, Kuntani N, Oka M. Acta Metall 1989;37(8):2223-8.

[6] Hayakawa M, Oka M. Acta Metall 1989;37(8):2229-35.

[7] Bateman CA, Notis MR. J Mater Sci Lett 1990;9(7):803-6.

[8] Shibata M, Kato M, Seto H, Noma T, Yoshimura M, Somiya S. J Mater Sci 1987;22:1432-6.

[9] Lee JH, Kim I, Hulbert DM, Jiang D, Mukherjee AK, Zhang X, Wang H. Acta Mater 2010;58(14):4891-9.

[10] Minor AM, Syed Asif SA, Shan Z, Stach EA, Cyrankowski E, Wyrobek TJ, Warren OL. Nat Mater 2006;5(9):697-702.

[11] Soer WA, Hosson JTMD, Minor AM, Morris Jr JW, Stach EA. Acta Mater 2004;52(20):5783-90.

[12] Shan ZW, Adesso G, Cabot A, Sherburne MP, Syed Asif SA, Warren OL, Chrzan DC, Minor AM, Alivisatos AP. Nat Mater 2008;7(12):947-52.

[13] Beaber A, Gerberich W. Nat Mater 2010;9(9):698-9.

[14] Deneen J, Mook W, Minor A, Gerberich W, Barry Carter C. J Mater Sci 2006;41(14):4477-83.

[15] Calvié E, Joly-Pottuz L, Esnouf C, Clément P, Garnier V, Chevalier J, Yves. J, Malchère A, Epicier T, Masenelli-Varlot K. J Eur Ceram Soc 2012.

[16] Calvié E, Joly-Pottuz L, Esnouf C, Douillard T, Gremillard L, Malchère A, Masenelli-Varlot K. Submitted to J Microsc.

[17] Beche A, Rouviere JL, Clement L, Hartmann JM. Appl Phys Lett 2009;95(12):123114-3.

[18] Giannuzzi LA, Stevie FA. Micron 1999;30(3):197-204.

[19] Stadelman P. JEMS Software. Ultramicroscopy 1987.

[20] Li J, Malis T, Dionne S. Mater Charact 2006;57(1):64-70.

[21] Esnouf C. Caractérisation Microstructurale des Matériaux, first ed. Lyon: LPPUR; 2011.

[22] Morniroli JP. Electron Diffraction Software. Lille; 1998.

[23] Morniroli JP. Interf Sci 1997;4(3):273-83.

[24] Igawa N, Ishii Y, Nagasaki T, Morii Y, Funahashi S, Ohno H. J Am Ceram Soc 1993;76(10):2673-6. 
[25] Boudias C, Monceau D. CaRInE crystallography Software. Compiègne; 1989.

[26] Hirsch PB, Howie A, Nicholson DW, Pashley DW, Whelan MJ. Electron microscopy of thin crystals. London: Butterworths; 1969.

[27] Houdellier F, Jacob D, Casanove MJ, Roucau C. Ultramicroscopy 2008;108(4):295-301.

[28] Chuvilin A, Kaiser U, de Robillard Q, Engelmann H-Jr. J Electron Microsc 2005;54(6):515-7.

[29] Deville S, Guénin G, Chevalier J. Acta Mater 2004;52(19):5709-21.

[30] Houdellier F, Altibelli A, Roucau C, Casanove MJ. Ultramicroscopy 2008;108(5):426-32.

\section{Figure captions}

Figure 1: TEM Observation of the 12Ce-TZP thin foil. a) Bright field image with one grain outlined by a dotted line; b) diffraction pattern of the outlined grain in a $[1 \overline{1} 0]$ zone axis; c) CBED diffraction pattern of the same grain obtained under a $25 \overline{44} 4$. orientation; d) a simulated drawing of the CBED diffraction pattern performed using JEMS software of the tetragonal structure. The triangle of experimental Bragg lines is represented by dotted lines (slightly shifted for clearer display)

Figure 2: TEM Observation of the 3Y-TZP thin foil. a) Bright field image of a grain outlined by a white dotted line; b) its associated diffraction pattern in a $[1728 \overline{24}|| ,15 \overline{5202}$, and [25997] zone axis for monoclinic, tetragonal 1 and tetragonal 2 phases respectively; c) the associated CBED pattern; d) a simulated CBED diffraction pattern of the monoclinic structure. The triangle of experimental Bragg lines is represented by dotted lines.

Figure 3: Sequence of CBED patterns. a), b), c), d) recorded on a grain on the 3Y-TZP thin foil. The three patterns correspond to three positions of the electron probe on the grain. The splitting of the $(14 \overline{3})_{\mathrm{t} 2}$ line can be observed depending on the position of the probe; e), f), g), h) sequence of CBED patterns recorded on the 12Ce-TZP during nanoindentation. A splitting of the $(103)_{t}$ line is observed. 
Figure 4: TEM observations of the 12Ce-TZP thin foil a) Bright field image with a monoclinic germ at the boundary between two tetragonal grains; b), c), d), e), f) Sequence of LACBED patterns in a $2 \overline{41} 1187$ zone axis recorded at the interface between the monoclinic germ and the tetragonal grain (t1).

Figure 5: Schematic representation of:

a) The existence of a distorted area (circled in dotted line) at the boundary between the tetragonal and monoclinic phases. This area could be a consequence of the phase transformation and a preparation of its propagation; $b$ ) the existence of a distorted area (circled in dotted line) created under stress. This area could be a nucleus of the phase transformation.

Gray circles represent the area illuminated by the probe (10 nm diameter). The schematic CBED pattern of each area is represented. 